\title{
Neuroendocrine and Behavioral Effects of Repetitive Transcranial Magnetic Stimulation in a Psychopathological Animal Model Are Suggestive of Antidepressant-like Effects
}

Martin E. Keck, M.D., Tobias Welt, B.Sc., Anke Post, M.D., Marianne B. Müller, M.D., Nicola Toschi, Ph.D., Alexandra Wigger, Ph.D., Rainer Landgraf, Ph.D., Florian Holsboer, M.D., Ph.D., and Mario Engelmann, Ph.D.

The neuroendocrine and behavioral effects of repetitive transcranial magnetic stimulation (rTMS) were investigated in two rat lines selectively bred for high and low anxiety-related behavior. The stimulation parameters were adjusted according to the results of accurate computerassisted and magnetic resonance imaging-based reconstructions of the current density distributions induced by rTMS in the rat and human brain, ensuring comparable stimulation patterns in both cases. Adult male rats were treated in two 3-day series under halothane anesthesia. In the forced swim test, rTMS-treatment induced a more active coping strategy in the high anxiety-related behavior rats only (time spent struggling; $332 \%$ vs. controls), allowing these animals to reach the performance of low anxiety-related behavior rats. In contrast, rTMS-treated low anxiety-related behavior rats did not change their swimming behavior. The development of active coping strategies in high anxiety-related behavior rats was accompanied by a significantly attenuated stress-induced elevation of plasma corticotropin and corticosterone concentrations. In summary, the behavioral and neuroendocrine effects of rTMS of frontal brain regions in high anxiety-related behavior rats are comparable to the effects of antidepressant drug treatment. Interestingly, in the psychopathological animal model repetitive transcranial magnetic stimulation induced changes in stress coping abilities in the high-anxiety line only.

[Neuropsychopharmacology 24:337-349, 2001] (C) 2001 American College of Neuropsychopharmcology. Published by Elsevier Science Inc.
KEY WORDS: HPA system; ACTH; Depression; Stress coping; Frontal brain; rTMS; emotionality

Several lines of evidence resulting from both preclinical (Fleischmann et al. 1995; Zyss et al. 1997) and clinical (George et al. 1999) studies suggest that repetitive tran-

From the Max Planck Institute of Psychiatry, Munich, Germany. Address correspondence to: Dr. Martin E. Keck, Max Planck Institute of Psychiatry, Kraepelinstr. 2-10, D-80804 Munich, Germany. Tel.: ++49-89-30622-314; Fax: ++49-89-30622-569; E-mail: keck@ mpipsykl.mpg.de

Received February 28, 2000; revised July 25, 2000; accepted August 3, 2000. scranial magnetic stimulation (rTMS) of left frontal brain regions might have antidepressant effects. Recently, it was shown that acute and subchronic rTMS treatments reduced the duration of immobility in rodents in the Porsolt swim test (Fleischmann et al. 1995; Zyss et al. 1997), a test that is thought to have predictive value for the efficacy of antidepressant drugs in humans (Porsolt et al. 1977; for a review, see Lucki 1997). Moreover, we recently demonstrated that long-term rTMS in rats has a neuroprotective effect both in vitro and in vivo (Post et al. 1999). In addition, we observed rTMS-induced changes in the expression of brain-derived neurotrophic factor (BDNF) and cholecystokinin simi- 
lar to those reported after antidepressant drug treatment and electroconvulsive seizures (Müller et al. 2000). These data suggest that a common molecular mechanism may underlie different antidepressant treatment strategies and that animal experiments might be helpful in understanding the neurobiological changes induced by rTMS, which still remain to be investigated. This approach becomes even more attractive in view of the still limited knowledge about the hypothesized linkage between psychiatric diseases such as depression and changes in the regulation of the hypothalamicpituitary-adrenocortical (HPA) system (Holsboer 1995; 2000). In psychiatric disorders such as major depression, a variety of changes in HPA system regulation have been demonstrated, among them defective negative feedback of the HPA system, basal hypercortisolemia, dexamethasone nonsuppression, and a paradoxical corticotropin-releasing hormone (CRH) stimulation of ACTH after dexamethasone pretreatment (for a review, see Holsboer 1995; Holsboer and Barden 1996). In this context, HPA system function has been suggested as a common denominator for clinically effective antidepressant treatment strategies (Holsboer and Barden 1996). Indeed, studies of antidepressant action in humans and rats have shown that chronic antidepressant drug treatment is accompanied by an attenuation of HPA system activity (Heuser et al. 1996; Reul et al. 1993, 1994). These findings are further supported by the observation that antidepressants lower the levels of $\mathrm{CRH}$ in the cerebrospinal fluid of depressed patients (De Bellis et al. 1993). Thus, the antidepressant-evoked normalization of HPA system control may be causally related to the clinical remission. Moreover, the possible role of the prefrontal cortex in the regulation of HPA function is of considerable interest, suggesting the existence of a neuronal circuit whereby cortical activity might regulate endocrine responses to stress (Diorio et al. 1993). As a non-invasive tool, rTMS is suitable for further exploration of these mechanisms.

To obtain predictions about the clinical condition in human depression, an animal model of depressive-like behavior with face validity should be used (Geyer and Markou 1995). A pragmatic approach is to identify those behaviors that are frequently disturbed in the clinical condition and that can be studied confidently in animals (e.g., Willner et al. 1992). Such behaviors include anxiety-related behavior and stress coping strategies. The present series of experiments was conducted to investigate the neuroendocrine and behavioral impact of rTMS of left frontal brain regions in an appropriate animal model that reflects significant psychopathological features of human depression. We therefore characterized the effects of rTMS on the regulation of HPA system activity, stress coping and anxiety-related behavior in two Wistar rat lines selectively bred for high (HAB) and low (LAB) anxiety-related behavior under a regimen adapted from clinical conditions. These two rat lines differ not only in their inborn anxiety, but also in their stress coping strategies, their HPA system susceptibility to external stressors and their reactivity to benzodiazepine treatment (Landgraf et al. 1999; Liebsch et al. 1998a). As a key part of the study, a series of computer-assisted, magnetic resonance imaging (MRI)-based reconstructions of the current density distributions induced by rTMS in the rat and human brain were performed. This enabled us to adapt the experimental setup to achieve an intracerebral current density distribution that is comparable to the one induced in patients during standard clinical treatment.

\section{METHODS}

\section{Animals}

The animal studies were conducted in accordance with the Guide for the Care and Use of Laboratory Animals of the Government of Bavaria, Germany, and according to the NIH guidelines. Experiments were carried out on adult ( $n=65 ; 358 \pm 19$ g body weight) male HAB and $\mathrm{LAB}$ rats. At the age of 10 weeks, prior to the experiments performed, $\mathrm{HAB}$ and $\mathrm{LAB}$ rats were tested on the elevated plus-maze (EPM) according to the breeding protocol to confirm the emotional trait (Landgraf et al. 1999; Liebsch et al. 1998a, 1998b). Rats were housed in groups of six in the breeding unit of the Max Planck Institute of Psychiatry under standard laboratory conditions (12:12 hr light:dark cycle with lights on at 0700, $22 \pm 1{ }^{\circ} \mathrm{C}, 60 \%$ humidity, pelleted food and water ad libitum). One day prior to the onset of rTMS, animals were separated and housed individually until the end of the experiments. In Experiment 1 we examined the effects of rTMS on anxiety-related and stress-coping behavior $(n=41)$. In Experiment 2 we examined the effects of rTMS on neuroendocrine reactivity to different stressors $(n=24)$. To control for the development of body weight, animals were weighed weekly during the experiments.

\section{Computer Modeling}

Background. rTMS relies on the principle that a timevarying magnetic induction field, commonly generated by passing strong current pulses through a coiled wire, will induce a current flow in any medium with nonzero conductivity, provided a portion of the magnetic flux is concatenated by the bound medium. In the case of rTMS, the medium is composed of biological tissue with highly inhomogeneous electrical characteristics, which under the influence of a dynamically varying magnetic induction field will give rise to inhomogeneous electrostatic field distributions. These contributions to the total electric field (and hence the induced 
current density distribution) resulting in the brain cannot be neglected, as they account for the influence of the brain's finite and inhomogeneous conductivity patterns. We therefore selected a theoretical and computational approach (Cerri et al. 1995) which allows for all relevant electromagnetic effects to be taken into account, achieving an accurate reconstruction of the conductive phenomena elicited by rTMS both in the rat and the human brain.

Model Structure. Briefly, the electrical characteristics of the brain were reconstructed from MRI images, whereby the conductivity distribution of the tissue was mapped onto the anatomical data. The map consisted of a fourth degree interpolating polynomial which takes the grayscale values (0-255) of the MRI images as the argument and returns the corresponding conductivity value at that point in space. The five known data points between which we interpolated were taken from the literature (Cerri et al. 1995; Ravazzani et al. 1996), where values ranged from $0.01 \mathrm{~A} / \mathrm{Vm}$ (bone) to $1.6 \mathrm{~A} / \mathrm{Vm}$ (cerebrospinal fluid). In between were skin, gray matter, and white matter. A 3D network consisting of cubic cells was then fitted to the brain, which for the order of magnitude of the electromagnetic parameters appropriate for rTMS can be considered to be purely resistive (Cerri et al. 1995; Ravazzani et al. 1996). The number of meshes was 100 in the $\mathrm{x}$ and $\mathrm{y}$ directions and 85 in the $\mathrm{z}$ direction since the vertical edge of the reconstructed solid was shorter. The resistivity value for each cell edge follows directly from the spatial conductivity map. Then, the magnetic induction field was calculated as a function of the coil geometry and the time evolution of the incoming current, after which discretised versions of Maxwell's equations in integral form were solved simultaneously for all meshes in the network. This allowed for an exact characterization of the total electric field and current density distributions arising in the brain during rTMS for arbitrary coil shapes, orientations and currents.

Model Implementation. Complete anatomical MRI scans were obtained for both human and rat brain. The routines for mapping electrical conductivity value to the biological tissue as well as for the calculation of the induction field and the solution of the resulting linear system were written in $\mathrm{C}++$ for faster execution. The coefficient matrix of the system was extremely sparse, so that especially designed routines could be recruited for its solution. Nevertheless, the dimensions of the system (between $10^{6}$ and $10^{7}$ unknowns, approximately $10^{7}-10^{8}$ non-zero matrix elements depending on the resolution used) made the employment of large scale computer resources unavoidable. A parallel version of the code was therefore implemented on a Cray T3E ma- chine (784 RISC CPUs, 100 GB RAM, Computing Center of the Max Planck Society, Garching).

\section{Repetitive Transcranial Magnetic Stimulation}

A commercially available stimulator $\left(\mathrm{MagPro}^{\mathrm{TM}}\right.$, DANTEC, Skovlunde, Denmark) was used for rTMS. The stimulus waveform was biphasic with a pulse width of $280 \mu \mathrm{s}$. The temperature of the stimulation coil $\left({ }^{\circ} \mathrm{C}\right)$ and stimulus intensity $(\mathrm{A} / \mu \mathrm{s})$ were monitored from a digital output display. A round prototype coil was selected (S60, DANTEC; inner diameter: $6 \mathrm{~mm}$; outer diameter: $57 \mathrm{~mm}$; 21 windings). The initial current direction was clockwise.

Each HAB and LAB rat received 1,000 stimuli/day resulting from 20 trains with a maximum magnetic induction field modulus of 4 Tesla [120 A/ $\mu$ s; $130 \%$ of rats' motor threshold, determined as described previously (Müller et al. 2000)] at a rate of $20 \mathrm{~Hz}$ for $2.5 \mathrm{~s}$. These stimulation parameters were chosen according to the results of accurate computer reconstructions as described above. Between trains there was an interval of 2 min for cooling to prevent overheating of the stimulation coil. The coil was held in direct physical contact to the animals' head at the stimulation point. To mimic clinical conditions (Pascual-Leone et al. 1996), the stimulation point was set at the left frontal cortex, as determined according to the atlas of Greene (1963). Due to direct muscular activation animals of the rTMS treatment group (Experiment 1: $\mathrm{HAB} n=11$; LAB $n=9$; Experiment 2: $\operatorname{HAB} n=6$; $\operatorname{LAB} n=6$ ) experienced a slight tremor of the head during stimulation. Control rats (Experiment 1: $\mathrm{HAB} n=11$; LAB $n=10$; Experiment 2: $\operatorname{HAB} n=6$; $\operatorname{LAB} n=6$ ) were exposed to the acoustic artifact (i.e., a clicking sound which is caused by the rapid expansion/contraction that the coiled conducting wire inside the coil experiences as a result of the dynamically varying, high intensity current) with the stimulation coil held perpendicularly $10 \mathrm{~cm}$ above the head. Animals were treated in two 3-day series separated by a 4-day interval. In a pilot study unrestrained, nonhandled, adult rats that were subjected to the same rTMS protocol as in the present study showed clear unspecific stress responses, as evidenced, for instance, by consecutive locomotor hyperactivity and aggressive behavior. Due to the breeding protocol adult rats had to be used, therefore excluding the possibility of adapting them to the stimulation procedure by an intensive training. All animals (rTMS treatment group and control rats) were therefore anesthetized throughout rTMS or sham treatment. The body temperature was measured with a thermistor in the colon and maintained at $37.5^{\circ} \mathrm{C}$ with an electric heating pad. Halothane was used as the inhalation anesthetic via a face mask to keep the rats sedated for the 1-hr period required for rTMS or sham treatment. Anesthesia was kept at a low level and the corneal reflex was checked regularly to be sure that it was preserved throughout anesthesia. 


\section{Experiments 1 and 2: Behavioral Testing}

All behavioral tests were videotaped so they would be available for re-scoring and re-analyzing.

Elevated Plus-maze (EPM) Test. Nine weeks after their initial EPM test and after two 3-day series of chronic rTMS-treatment, $\mathrm{HAB}$ and $\mathrm{LAB}$ rats were tested on the EPM. The EPM was made of dark gray PVC and consisted of a plus-shaped platform elevated $73 \mathrm{~cm}$ from the floor. Two of the opposing arms $(50 \times 10 \mathrm{~cm})$ were enclosed by $38 \mathrm{~cm}$ high side and end walls (closed arms). The other two arms had no walls (open arms). All four arms were connected at the center by a $10 \times 10$ $\mathrm{cm}$ square platform. This test has been validated for the detection of emotional responses to anxiogenic and anxiolytic substances (Pellow et al. 1985) as well as for the determination of inborn emotionality (Liebsch et al. 1998a, 1998b). Briefly, it is based on the creation of a conflict between the exploratory drive of the rat and its innate aversion to open, exposed and elevated areas. Thus, reduced open-arm exploration serves as a measure of increased anxiety. Rats' behavior during $5 \mathrm{~min}$ was monitored via a video camera mounted above the platform. Measures were (1) entries into the open arms (expressed as the percentage of all arm entries that were open arm entries; an entry was counted when the rat had placed both forepaws into the respective arm), (2) time spent on the open arms (expressed as the percentage of time spent on all arms that was spent on open arms), (3) latency until first open-arm entry, (4) number of full (whole body) entries into the open arm, and (5) overall activity, measured as number of entries into the closed arms.

Forced Swim Test. HAB and LAB rats were first tested one day prior to the first rTMS treatment. The second test took place after completion of the rTMS protocol, 90 min after the elevated plus-maze testing. On the day of testing, between 0800 and 1200, each rat was placed for 5 min into a cylindrical Plexiglas tank $(40 \mathrm{~cm}$ high and 18 $\mathrm{cm}$ in diameter), filled with tap water $\left(23^{\circ} \mathrm{C}\right)$ up to a level of $25 \mathrm{~cm}$. During each swimming session, the following behaviors were scored by a trained observer by pressing pre-set keys on a personal computer (PC): (1) time spent struggling, defined as strongly moving all four limbs, with the front paws breaking the water surface; (2) time spent swimming, defined as moving all four limbs, swimming around in the tank or actively diving; (3) time spent floating, defined as remaining immobile with only occasional slight movements to keep the body balanced and the nose above water; and (4) latency until the first floating reaction.

\section{Experiment 2: Surgery}

rTMS-treated $\operatorname{HAB}(n=6)$ and LAB $(n=6)$ animals as well as the respective sham-treated animals of both lines
( $n=6$ each) underwent surgery one day prior to the second 3-day stimulation series. Surgery was performed under halothane anesthesia using aseptic procedures. The jugular vein was chronically catheterized for subsequent blood sampling. The catheter was exteriorized at the neck of the animal and filled with sterile saline containing gentamicin (30,000 IU/rat; Centravet, Bad Bentheim, Germany); $0.2 \mathrm{ml}$ were infused into the animal.

\section{Experiment 2: Blood Sampling}

Effects of Long-term rTMS on HPA System Activity in $H A B$ and LAB Rats. To monitor the release of ACTH and corticosterone into blood under basal conditions and in response to a predominantly emotional stressor (5 min EPM exposure) and a combined physical/emotional stressor ( $5 \mathrm{~min}$ forced swimming), blood samples were taken from both rTMS-treated and control freely moving HAB and LAB rats, beginning 5 days after surgery $(5,15,60 \mathrm{~min}$ after stress). Briefly, on each of the experimental days at 0700 the jugular venous catheter was connected via 50-cm PE-50 tubing to a plastic syringe filled with sterile heparinized saline $(30 \mathrm{IU} / \mathrm{ml}$; Ratiopharm, Ulm, Germany). Thereafter, the rats remained undisturbed for $2 \mathrm{hr}$ to adapt to the equipment.

Stimulation with i.v. CRH. On the following day at 0700 , the rats were weighed. Then the catheter was reconnected to a syringe filled with heparinized saline, and at 0900 a basal blood sample was taken $(0.2 \mathrm{ml}$ for ACTH and corticosterone). A further blood sample replaced by sterile saline was collected $10 \mathrm{~min}$ after i.v. bolus injection of human CRH $(50 \mathrm{ng} / \mathrm{kg}, 0.5 \mathrm{ml} / \mathrm{kg}$; Ferring, Kiel, Germany).

\section{Treatment of Blood Samples and RIAs}

Blood samples were collected in prechilled tubes containing EDTA and a protease inhibitor $(10 \mu l$ aprotinin, Trasylol $^{\mathrm{TM}}$, Bayer, Germany) and centrifuged (5 min, $4000 \mathrm{rpm}, 4^{\circ} \mathrm{C}$ ). The respective plasma samples were stored at $-80^{\circ} \mathrm{C}$ until measurement. Plasma ACTH (50 $\mu \mathrm{l})$ and corticosterone $(10 \mu \mathrm{l})$ levels were measured using commercially available kits (Biochem, Freiburg, Germany) according to the respective protocols (Sensitivity: ACTH: $<1.0 \mathrm{pg} / \mathrm{ml}$; corticosterone: $<2.0 \mathrm{ng} / \mathrm{ml}$ ). The intra- and interassay coefficients of variation were $7 \%$ and $10 \%$, respectively.

\section{Postmortem Analysis}

Animals were killed with an overdose of halothane at the end of the experiments. In all animals, the pituitary, adrenals and other internal organs were visually examined to exclude animals with tumors from the statistical analysis. 


\section{A}
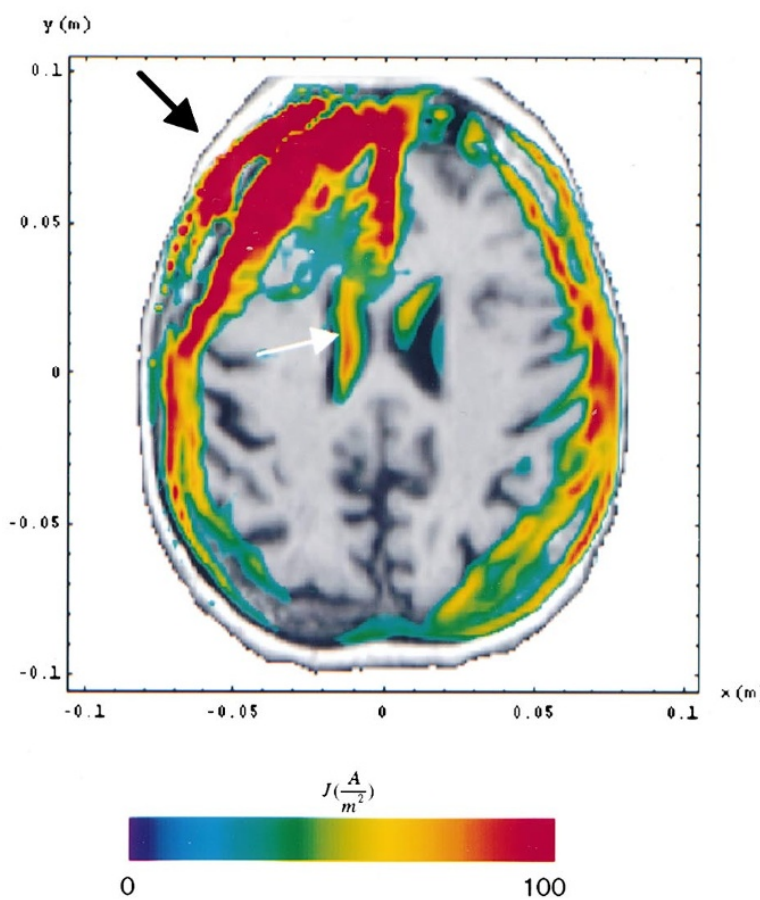

\section{C}

$y(m)$

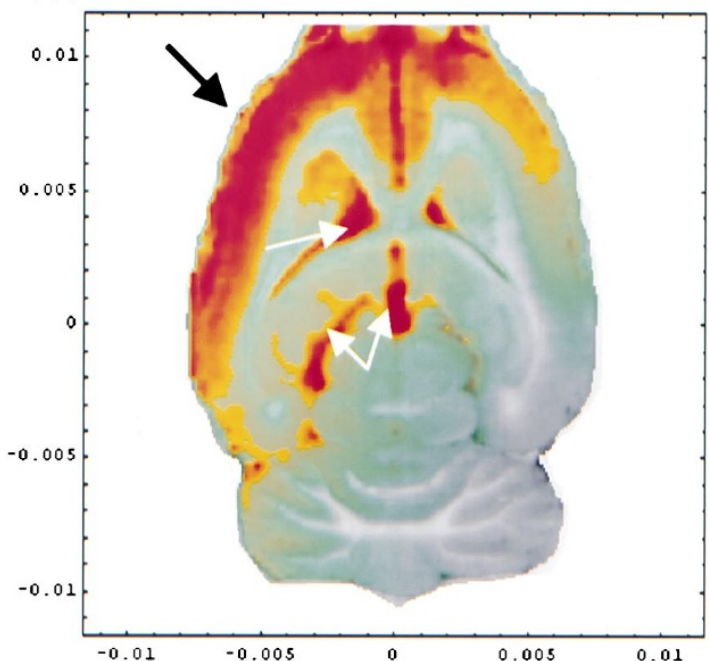

B

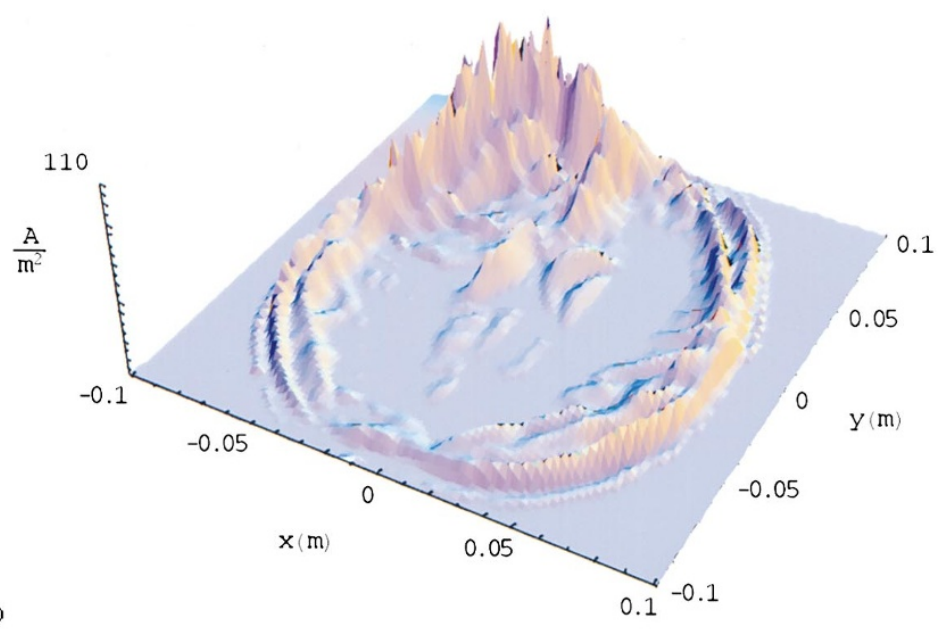

D

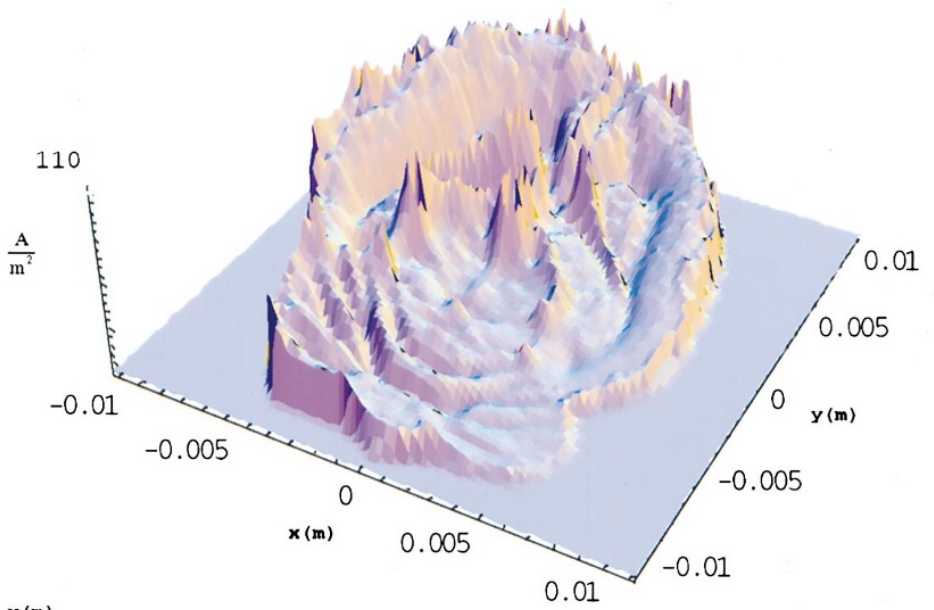

$\mathbf{x}(\mathrm{m})$

Figure 1. Spatial distribution of current density induced in one transversal layer of $(\mathbf{A}, \mathbf{B})$ human and $(\mathbf{C}, \mathbf{D})$ rat brain by rTMS. Density plot $(\mathbf{A}, \mathbf{C})$ and three-dimensional (B, D) representations. The electrical characteristics of the brain are reconstructed from MRI images, whereby the conductivity distribution of the tissue is mapped onto the anatomical map of the brain. Human: Maximum coil current intensity $\mathrm{I}_{\max }=4000$ A. Pulse rise time: approximately $60 \mu$ s. Commercial "Figure 8 " coil (DANTEC, Skovlunde, Denmark), inner diameter (ID) $20 \mathrm{~mm}$; outer diameter (OD) $100 \mathrm{~mm}$; 10 windings per coil. Resulting maximum magnetic induction modulus close to coil centre: approximately 1.8 Tesla. Transversal section: 49 mm from vertex. Coil center is at the same height as the section shown. Coil is tilted by $45 \%$ in the xy and yz planes (clinical use; see e.g. George et al. 1999; Pascual-Leone et al. 1996). Contact point is shown by black arrow (left dorsolateral prefrontal cortex). Average current density over red region: $92 \pm 1.5 \mathrm{~A} / \mathrm{m}^{2}$. White arrow indicates cerebrospinal fluid (CSF) with highest conductivity value (1.6 A/Vm). Rat: $\mathrm{I}_{\max }=6000$ A. Pulse rise time: approx. $60 \mu$ s. Prototype round coil, ID: 6 mm, OD: 57 $\mathrm{mm}, 21$ windings (DANTEC). Resulting maximum magnetic induction modulus close to coil edge: approximately 4.1 Tesla. Transversal section: $1.5 \mathrm{~mm}$ from vertex. Coil center is at the same height as the section shown. Coil is tilted by $45 \%$ in the xy and yz planes. Contact point is shown by black arrow (left prefrontal cortex). Average current density over red region: $97 \pm$ $0.6 \mathrm{~A} / \mathrm{m}^{2}$. White arrow indicates CSF. 


\section{Statistics}

Results are presented as means \pm SEM. Statistical analysis was performed with a software package (GBStat version 6.0, Dynamic Microsystems, Silver Spring, MD). Statistical significance for plasma ACTH and corticosterone levels was determined between the groups using two-way analysis of variance (ANOVA) for repeated measures (group $\times$ time) and within the groups by one-way ANOVA for repeated measures. Analysis of the behavioral parameters was carried out using a three-way ANOVA (line $\times$ treatment $\times$ time) with repeated measures on the last factor (EPM and forced swim test). When appropriate, a post hoc Newman-Keuls test was used. Delta values (maximum post-stress minus baseline levels) for each stress condition were calculated and compared using the MannWhitney U-test. $p<.05$ was considered statistically significant.

\section{RESULTS}

All experimental animals were included in the statistical analysis as postmortem visual examination failed to reveal any organ pathologies. The weight gain of the rats, controlled weekly, was indistinguishable between groups (data not shown).

\section{Computer Modeling}

Figure 1 shows the predicted current density distributions induced by rTMS in one transversal layer of the rat and human brain. The stimulation parameters (see legend) for the human brain are adjusted to the most commonly used setup for clinical applications. The values of these parameters for stimulation of the rat brain correspond to those adopted in our experimental setup. It is shown that it is possible to elicit comparable effects in the rat and human brain.

\section{Forced Swim Test}

Experiment 1. A three-way ANOVA (line $\times$ treatment $\times$ time) with repeated measures on the last factor re- vealed a significant line difference in all parameters measured before and after rTMS treatment except swimming time (time spent struggling: $\mathrm{F}_{1,38}=59.82$, $p<.0001$; floating: $\mathrm{F}_{1,38}=357.9, p<.0001$; latency to first floating: $F_{1,38}=78.20, p<.0001$ ), with a significant interaction with factor treatment for time spent struggling $\left(\mathrm{F}_{1,38}=16.41, p=.0002\right)$ and floating $\left(\mathrm{F}_{1,38}=\right.$ $31.18, p<.0001)$. There was also a significant interaction between the factors time and treatment for time spent struggling $\left(\mathrm{F}_{1,38}=29.39, p<.0001\right)$ and floating $\left(\mathrm{F}_{1,38}=48.12, p<.0001\right)$. A significant interaction of all three factors was found only for the time spent floating $\left(\mathrm{F}_{1,38}=32.04, p<.0001\right)$. Post hoc analysis showed that the time spent struggling and the latency until the first floating reaction in rTMS-treated $\mathrm{HAB}$ rats was no longer different from the LAB rats (Table 1). The rTMStreated HAB animals showed significantly more struggling $(p<.01$; Table 1$)$ than the sham-stimulated HAB controls but floated less $(p<.01$; Table 1$)$. The latency until the first floating reaction was significantly longer in the rTMS-treated rats than in the controls $(p<.01$; Table 1).

Experiment 2 (with Simultaneous Blood Sampling). A three-way ANOVA (line $\times$ treatment $\times$ time) with repeated measures on the last factor revealed a significant line difference in all parameters measured before and after rTMS treatment except swimming time (time spent struggling: $\mathrm{F}_{1,20}=21.23, p=.0003$; floating: $\mathrm{F}_{1,20}=$ $158.8, p<.0001$; latency to first floating: $\mathrm{F}_{1,20}=19.89$, $p=.0004)$, with a significant interaction with factor treatment for time spent struggling $\left(\mathrm{F}_{1,20}=5.97, p=\right.$ $.0265)$ and floating $\left(\mathrm{F}_{1,20}=19.39, p=.0004\right)$. There was also a significant interaction between the factors time and treatment for time spent struggling $\left(\mathrm{F}_{1,20}=21.96\right.$, $p=.0002)$ and floating $\left(\mathrm{F}_{1,20}=68.12, p<.0001\right)$. A significant interaction of all three factors was found only for the time spent floating $\left(\mathrm{F}_{1,20}=39.73, p<.0001\right)$. Post hoc analysis showed that the time spent struggling and floating and the latency until the first floating reaction in rTMS-treated $\mathrm{HAB}$ rats was no longer different from the LAB rats (Figure 2). The rTMS-treated HAB animals showed significantly more struggling $(332 \%, p<.01$;

Table 1. Forced Swim Behavior of rTMS-treated and Sham-treated Male HAB and LAB Rats

\begin{tabular}{|c|c|c|c|c|c|c|}
\hline & LAB (pre-treatment) & LAB (sham) & LAB (rTMS) & НАВ (pre-treatment) & НАВ (sham) & НАВ (rTMS) \\
\hline Struggling (sec) & $197 \pm 17^{* *}$ & $150 \pm 19^{* *}$ & $162 \pm 18$ & $73 \pm 5$ & $48 \pm 10$ & $160 \pm 13^{+}$ \\
\hline Floating (sec) & $12 \pm 3.3^{* *}$ & $19 \pm 5^{* *}$ & $4 \pm 1^{*}$ & $98 \pm 5$ & $145 \pm 15$ & $23 \pm 5^{+}$ \\
\hline Swimming (sec) & $94 \pm 8$ & $136 \pm 15$ & $135 \pm 18$ & $130 \pm 11$ & $109 \pm 14$ & $111 \pm 12$ \\
\hline Latency (sec) & $176 \pm 17^{* *}$ & $90 \pm 28^{* *}$ & $76 \pm 21$ & $5 \pm 2$ & $11 \pm 3$ & $58 \pm 15^{\dagger}$ \\
\hline
\end{tabular}

Forced swim behavior: Time (sec) spent struggling, floating, or swimming and latency (sec) until the first floating reaction of male high (HAB; rTMS: $n=11$; sham: $n=11$ ) and low (LAB; rTMS: $n=9$; sham: $n=10)$ anxiety-related behavior rats during a 5 -min swim session (23 ${ }^{\circ}$ ). HAB and $\mathrm{LAB}$ rats were first tested one day prior to the first rTMS treatment (pre-treatment). The second test took place after completion of the rTMS protocol. Data are means \pm SEM. ${ }^{*} p<.05,{ }^{* *} p<.01$ vs. HAB rats; ${ }^{\dagger} p<.01$ vs. pre-treatment and sham in HAB rats. 

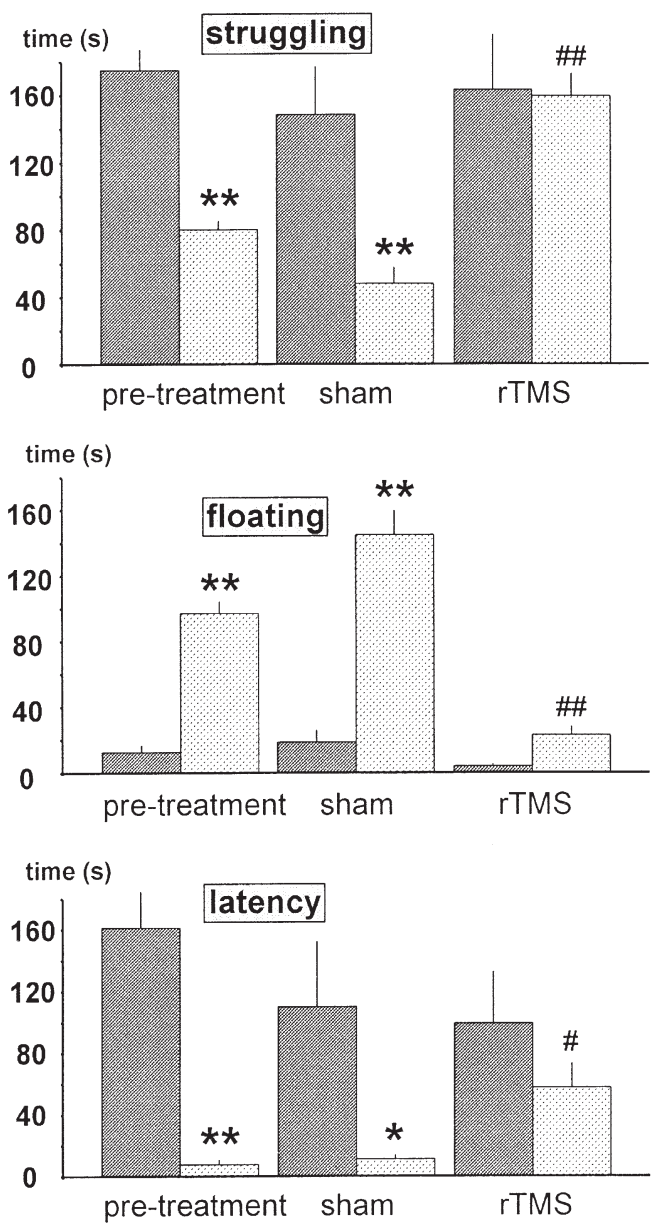

Figure 2. Behavioral measures obtained in the forced swim test, performed prior to (pre-treatment) and after completion of the rTMS protocol in LAB (rTMS: $n=6$; sham: $n=6$; gray bars) and HAB (rTMS: $n=6$; sham: $n=6$; shaded bars) rats. Time during the 5-min testing session spent struggling and floating, respectively, and latency until the first floating reaction. ACTH data shown in Figure 3 were measured simultaneously. Data are means + SEM. ${ }^{*} p<.05,{ }^{* *} p<.01$ vs. LAB; $\# p<.05, \# \#<.01$ vs. pre-treatment and sham in $\mathrm{HAB}$ rats.

Figure 2) than the sham-stimulated $\mathrm{HAB}$ controls $(100 \%)$ but floated less $(16 \%, p<.01$; Figure 2$)$. The latency until the first floating reaction was significantly longer in the rTMS-treated rats than in the controls $(526 \%, p<.01$; Figure 2$)$.

\section{Elevated Plus-maze Test}

Experiment 1. A three-way ANOVA (line $\times$ treatment $\times$ time) with repeated measures on the last factor on the main EPM parameters confirmed that $\mathrm{HAB}$ rats displayed a lower percentage of entries into $\left(\mathrm{F}_{1,38}=64.20\right.$, $p<.0001$; Table 2) and time spent on $\left(\mathrm{F}_{1,38}=135.9, p<\right.$ .0001 ; Table 2) the open arms of the EPM than the ani-
Table 2. Elevated Plus-maze Performance of rTMS-treated and Sham-treated Male HAB and LAB Rats

\begin{tabular}{lcc}
\hline & \multicolumn{2}{c}{ EPM (\%) } \\
\cline { 2 - 3 } & rTMS & Sham \\
\hline Time on open arms; LAB rats & $23.7 \pm 3.3^{* *,+}$ & $42.4 \pm 5.9^{* *}$ \\
Time on open arms; HAB rats & $6.3 \pm 4.0$ & $3.6 \pm 2.3$ \\
Entries into open arms; LAB rats & $33.0 \pm 3.1^{* *}$ & $45.9 \pm 2.3^{* *}$ \\
Entries into open arms; HAB rats & $7.7 \pm 4.6$ & $14.5 \pm 5.8$ \\
\hline
\end{tabular}

Elevated plus-maze (EPM; percentage of time spent on and number of entries into the open arms compared to the total for all arms) performance of male high (HAB; rTMS: $n=6$; sham: $n=6$ ) and low (LAB; rTMS: $n=6$; sham: $n=6$ ) anxiety-related behavior rats after two 3-day series of rTMS treatment. Data are means \pm SEM. ${ }^{* *} p<.01 \mathrm{vs}$. HAB rats; ${ }^{+} p<.01$ vs. sham treatment.

mals from the LAB line both before and after rTMS treatment. Accordingly, the latency until the first openarm entry was much higher in HAB (rTMS: $200 \pm 37 \mathrm{~s}$, control: $252 \pm 32 \mathrm{~s}$ ) than in LAB (rTMS: $72.8 \pm 22 \mathrm{~s}$, control: $\left.42.3 \pm 12 \mathrm{~s} ; \mathrm{F}_{1,38}=36.02, p<.0001\right)$ rats. Overall activity, indicated by the number of closed-arm entries, failed to show a significant difference between $\mathrm{HAB}$ (rTMS: $2.8 \pm 1.1$, control: $4.3 \pm 1.0$ ) and LAB (rTMS: $5.8 \pm 1.2$, control: $5.3 \pm 0.6$ ) animals. A change over time was detected for the percentage of time spent on the open arms $\left(\mathrm{F}_{1,38}=23.77, p<.0001\right)$; there were significant interactions with the factors line $\left(\mathrm{F}_{1,38}=23.67\right.$, $p<.0001)$ and treatment $\left(\mathrm{F}_{1,38}=7.77, p<.0001\right)$. A significant interaction of all three factors was found for the percentage of entries into $\left(\mathrm{F}_{1,38}=5.25, p=.0276\right)$ and time spent on the open arms $\left(\mathrm{F}_{1,38}=8.37, p=.0063\right)$. Post hoc comparison revealed that rTMS treatment in $\mathrm{LAB}$ rats resulted in a decrease in the percentage of time spent on the open arms $(p<.01$; Table 2$)$. However, the line differences between $\mathrm{HAB}$ and $\mathrm{LAB}$ rats were still present $(p<.01$; Table 2$)$.

Experiment 2 (with Simultaneous Blood Sampling). A three-way ANOVA (line $\times$ treatment $\times$ time) with repeated measures on the last factor on the main EPM parameters confirmed that $\mathrm{HAB}$ rats displayed a lower percentage of entries into $\left(\mathrm{F}_{1,20}=526.1, p<.0001\right)$ and time spent on $\left(\mathrm{F}_{1,20}=184.1, p<.0001\right)$ the open arms of the EPM than the animals from the LAB line both before and after rTMS treatment (data not shown). Accordingly, the latency until the first open-arm entry was higher in HAB (rTMS: $219 \pm 50 \mathrm{~s}$, control: $300 \pm 0 \mathrm{~s}$ ) than in LAB (rTMS: $106 \pm 23 \mathrm{~s}$, control: $62.3 \pm 20 \mathrm{~s}$; $\left.\mathrm{F}_{1,20}=131.3, p<.01\right)$ rats. Overall activity, indicated by the number of closed-arm entries, failed to show a significant difference between HAB (rTMS: $3 \pm 0.6$, control: $2.8 \pm 1.6$ ) and LAB (rTMS: $5.2 \pm 1.2$, control: $4.8 \pm$ 0.4 ) animals. A significant interaction of all three factors was found for the percentage of entries into $\left(\mathrm{F}_{1,20}=\right.$ $7.02, p=.0175)$ and time spent on the open arms $\left(\mathrm{F}_{1,20}=\right.$ 
Table 3. Plasma ACTH and Corticosterone (Cort) Concentrations in Response to Elevated Plus-maze Exposure (5 min) in rTMS-treated and Sham-treated Male HAB and LAB Rats

\begin{tabular}{|c|c|c|c|c|c|c|}
\hline \multirow[b]{2}{*}{ Line } & \multicolumn{6}{|c|}{ Elevated Plus-Maze } \\
\hline & Basal 1 & Basal 2 & +5 Min & +15 Min & $+60 \mathrm{Min}$ & Delta \\
\hline \multicolumn{7}{|l|}{ LAB, sham } \\
\hline $\mathrm{ACTH}(\mathrm{pg} / \mathrm{ml})$ & $33.0 \pm 3.3$ & $33.9 \pm 5.2$ & $90.4 \pm 14.5$ & $86.5 \pm 12.9$ & $57.3 \pm 11.3$ & $57.4 \pm 11.2$ \\
\hline Cort (ng/ml) & $24.6 \pm 11.5$ & $16.5 \pm 2.3$ & - & $246.9 \pm 7.7$ & $81.1 \pm 13.2$ & $230.4 \pm 5.4$ \\
\hline \multicolumn{7}{|l|}{ LAB, rTMS } \\
\hline $\mathrm{ACTH}(\mathrm{pg} / \mathrm{ml})$ & $38.6 \pm 5.0$ & $43.6 \pm 11.5$ & $200.1 \pm 52.3^{*}$ & $174.1 \pm 37.8^{*}$ & $52.6 \pm 11.6$ & $161.5 \pm 47^{*}$ \\
\hline Cort $(\mathrm{ng} / \mathrm{ml})$ & $43.5 \pm 15.1$ & $46.4 \pm 24.6$ & - & $264.5 \pm 34.2$ & $94.6 \pm 23.7$ & $221 \pm 19.1$ \\
\hline \multicolumn{7}{|l|}{ HAB, sham } \\
\hline $\mathrm{ACTH}(\mathrm{pg} / \mathrm{ml})$ & $33.0 \pm 3.6$ & $41.8 \pm 9.5$ & $210 \pm 27.9$ & $276.5 \pm 42.2$ & $96.2 \pm 11.3$ & $243.5 \pm 39$ \\
\hline Cort $(\mathrm{ng} / \mathrm{ml})$ & $22.9 \pm 15.4$ & $15.1 \pm 6.3$ & - & $325.8 \pm 15.1$ & $186.9 \pm 36.5$ & $310.7 \pm 4.3$ \\
\hline \multicolumn{7}{|l|}{ HAB, rTMS } \\
\hline $\mathrm{ACTH}(\mathrm{pg} / \mathrm{ml})$ & $26.1 \pm 1.9$ & $32.5 \pm 3.0$ & $130.5 \pm 25.1^{*}$ & $159.1 \pm 24.2^{*}$ & $47.7 \pm 3.0$ & $133 \pm 22.3^{*}$ \\
\hline Cort (ng/ml) & $19.4 \pm 6.3$ & $13.8 \pm 3.5$ & - & $241.3 \pm 16.2^{*}$ & $111.9 \pm 22.4^{*}$ & $227.5 \pm 12.7^{*}$ \\
\hline
\end{tabular}

$n=6$ per group. Data are means \pm SEM. ${ }^{*} p<.01$ vs. sham.

$6.32, p=.0229)$. Post hoc comparison revealed that rTMS treatment in LAB rats resulted in a decrease in the percentage of time spent on the open arms (rTMS: $22.8 \pm$ 1.2 , control: $34.3 \pm 1.3 ; p<.01)$. However, the line differences between $\mathrm{HAB}$ and $\mathrm{LAB}$ rats were still present $(p<.01)$.

\section{Effects of Long-term rTMS on HPA System Activity}

Effects of EPM Exposure in HAB Rats. Basal plasma ACTH and corticosterone levels were comparable in the rTMS-treated and control rats (Table 3). Exposure to the EPM caused a significant increase in plasma ACTH (two-way ANOVA, factor: time; $\mathrm{F}_{4,40}=47.13$, $p<.0001$ ) and corticosterone (two-way ANOVA, factor: time; $\left.\mathrm{F}_{3,30}=103.9, p<.0001\right)$ in both treatment groups. Two-way ANOVA revealed a significant interaction with the factor treatment (ACTH: $\mathrm{F}_{4,40}=3.70, p=$ .0138; corticosterone: $\left.\mathrm{F}_{3,30}=3.29, p=.0339\right)$. As shown in Figure 3A, the increase in plasma ACTH levels $5 \mathrm{~min}$ after EPM exposure was significantly lower in the rTMS-treated than in the control HAB rats $(p<.01)$. Similarly, the increase in plasma ACTH and corticosterone levels $15 \mathrm{~min}$ after EPM exposure was significantly lower in the rTMS-treated than in the control HAB rats $(p<.01)$. In contrast to corticosterone $(p<$ .01 ), plasma ACTH levels 60 min after EPM exposure were statistically indistinguishable between the two groups (Table 3).

Figure 3. (A) Effects of 5-min exposure to the elevated plus-maze (EPM) and forced swimming (FS) on plasma ACTH in HAB rats: rTMS-treated animals $(n=6$; black squares) and sham-treated controls $(n=6$; shaded squares;

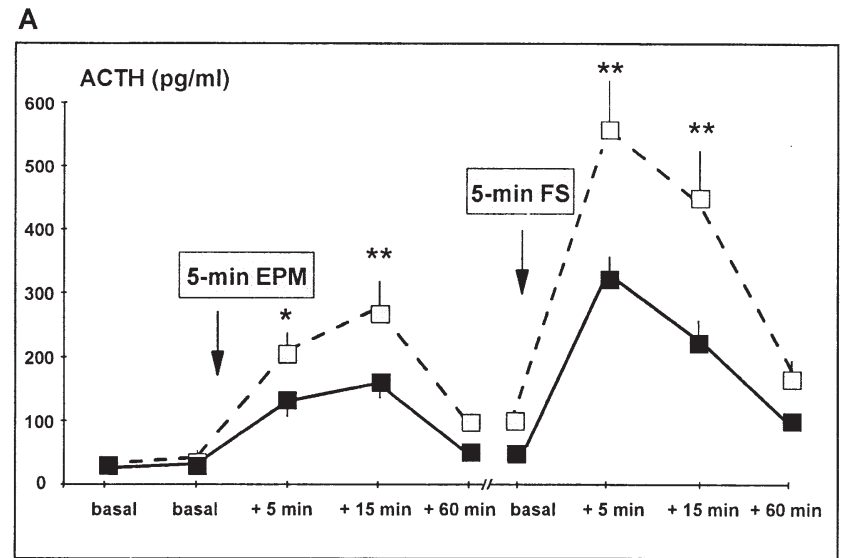

B

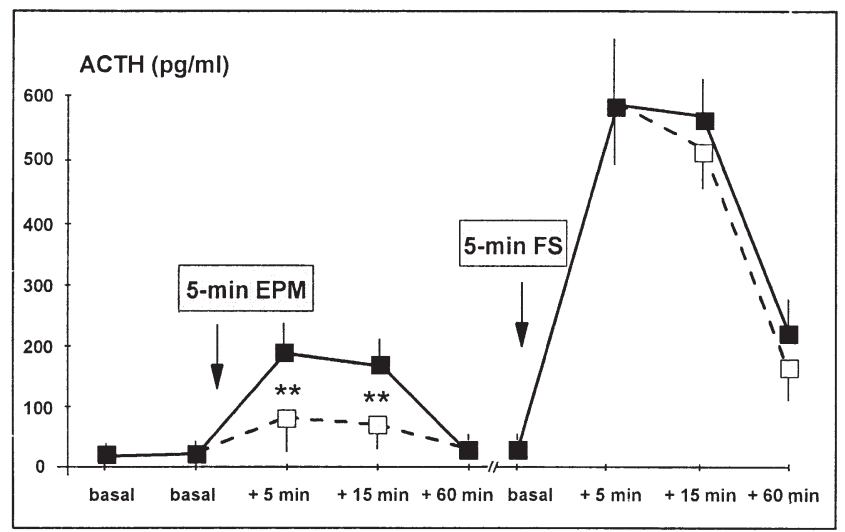

broken line). Corresponding behavioral data are shown in Figure 2. Data are means + SEM. ${ }^{* *} p<.01 ;{ }^{*} p<.05$ vs. control. (B) Effects of 5-min exposure to the elevated plus-maze (EPM) and forced swimming (FS) on plasma ACTH in LAB rats: rTMS-treated animals ( $n=6$; black squares) and shamtreated controls ( $n=6$; shaded squares; broken line). Corresponding behavioral data are shown in Figure 2. Data are means + SEM. ${ }^{* *} p<.01$ vs. control. 
Effects of EPM Exposure in LAB Rats. Basal plasma ACTH and corticosterone levels were comparable in the rTMS-treated and control rats (Table 3). For ACTH two-way ANOVA revealed a significant interaction between the factors group and time $\left(\mathrm{F}_{4,40}=3.97, p=.01\right)$. As shown in Figure 3B, the increase in plasma ACTH levels $5 \mathrm{~min}$ after EPM exposure was significantly higher in the rTMS-treated than in the control LAB rats $(p<.01)$. Similarly, the increase in plasma ACTH levels 15 min after EPM exposure was significantly higher in the rTMS-treated than in the control LAB rats $(p<.01)$. Plasma ACTH levels 60 min after EPM exposure were statistically indistinguishable between the two groups. There were no statistically significant differences in corticosterone release (Table 3).

Effects of Forced Swimming in HAB Rats. Basal plasma $\mathrm{ACTH}$ and corticosterone levels were taken $60 \mathrm{~min}$ after EPM exposure (Table 4). Forced swimming caused a significant increase in plasma ACTH (two-way ANOVA, factor: time; $F_{3,30}=60.66, p<.0001$ ) and corticosterone $\left(\mathrm{F}_{2,20}=103.8, p<.0001\right)$ in both treatment groups. For ACTH, two-way ANOVA revealed a significant interaction with the factor treatment $\left(\mathrm{F}_{3,30}=5.47\right.$, $p=.0052)$. As shown in Figure 3A, the increase in plasma ACTH levels 5 min after forced swimming was significantly lower in the rTMS-treated than in the control HAB rats $(p<.01)$. Similarly, the increase in plasma ACTH $(p<.01)$ and corticosterone $(p<.05)$ levels 15 min after forced swimming was significantly lower in the rTMS-treated than in the control HAB rats. In contrast, plasma ACTH and corticosterone levels $60 \mathrm{~min}$ after forced swimming were statistically indistinguishable between the two groups (Table 4 ).
Effects of Forced Swimming in LAB Rats. Basal plasma ACTH and corticosterone levels were taken $60 \mathrm{~min}$ after EPM exposure (Table 4). Forced swimming caused a significant increase in plasma ACTH $\left(\mathrm{F}_{3,30}=16.00, p<\right.$ $.0001)$ and corticosterone $\left(\mathrm{F}_{2,20}=238.8, p<.0001\right)$ in both treatment groups. The increase in plasma ACTH and corticosterone levels 5 and $15 \mathrm{~min}$ after forced swimming was similar in the rTMS-treated and control $\mathrm{LAB}$ rats. Furthermore, plasma ACTH and corticosterone levels $60 \mathrm{~min}$ after forced swimming were similar in the two groups (Table 4).

Effects of Stimulation with i.v. CRH. Basal plasma ACTH and corticosterone levels were comparable in the rTMS-treated and control HAB and LAB rats (Table $5)$. Infusion of $\mathrm{CRH}$ caused a significant increase in plasma ACTH and corticosterone in all groups $(p<$ .05). The increase in plasma ACTH and corticosterone levels $10 \mathrm{~min}$ after infusion of CRH was similar in the rTMS-treated and control HAB and LAB rats (Table 5).

\section{DISCUSSION}

This study provides the first evidence that long-term rTMS of frontal brain regions in psychopathological model animals induces (1) profound changes in acute stress coping strategies and (2) a concomitant attenuated response of the HPA system to stress. In HAB rats with a rather passive innate stress coping strategy, rTMS of left frontal brain regions markedly increased active coping, rendering these animals indistinguishable from LAB rats. No rTMS-induced changes were found in the anxiety-related behavior of HAB animals,

Table 4. Plasma ACTH and Corticosterone (Cort) Concentrations in Response to Forced Swimming $\left(5 \mathrm{~min} ; 23^{\circ} \mathrm{C}\right.$ ) in rTMS-treated and Sham-treated Male HAB and LAB Rats

\begin{tabular}{lccccc}
\hline & \multicolumn{5}{c}{ Forced Swimming } \\
\cline { 2 - 5 } Line & Basal & $+\mathbf{5}$ Min & $+\mathbf{1 5}$ Min & $+\mathbf{6 0}$ Min & Delta \\
\hline LAB, sham & & & \\
$\quad$ ACTH $(\mathrm{pg} / \mathrm{ml})$ & $57.3 \pm 11.3$ & $595 \pm 113$ & $518 \pm 55.0$ & $163 \pm 13.2$ & $537 \pm 101$ \\
$\quad$ Cort $(\mathrm{ng} / \mathrm{ml})$ & $57.2 \pm 9.7$ & - & $431 \pm 28.4$ & $402 \pm 33.8$ & $374 \pm 19$ \\
LAB, rTMS & & & & \\
$\quad$ ACTH $(\mathrm{pg} / \mathrm{ml})$ & $52.6 \pm 11.6$ & $590 \pm 104$ & $572 \pm 64$ & $221 \pm 56$ & $537 \pm 92$ \\
Cort $(\mathrm{ng} / \mathrm{ml})$ & $65.6 \pm 11.1$ & - & $434 \pm 13.5$ & $406 \pm 15.9$ & $368 \pm 5.2$ \\
HAB, sham & & & & \\
$\quad$ ACTH $(\mathrm{pg} / \mathrm{ml})$ & $66.2 \pm 9.3$ & $566 \pm 79$ & $469 \pm 84$ & $154 \pm 35$ & $403 \pm 74$ \\
Cort $(\mathrm{ng} / \mathrm{ml})$ & $54.8 \pm 8.8$ & - & $494 \pm 36.8$ & $339 \pm 47.9$ & $440 \pm 28$ \\
HAB, rTMS & & & & \\
ACTH $(\mathrm{pg} / \mathrm{ml})$ & $47.7 \pm 3.0$ & $323 \pm 25^{* *}$ & $238 \pm 28^{* *}$ & $99 \pm 4.3$ & $275 \pm 23^{* *}$ \\
Cort $(\mathrm{ng} / \mathrm{ml})$ & $69.7 \pm 11.9$ & - & $422 \pm 30.8^{*}$ & $281 \pm 19.8$ & $352 \pm 19^{*}$ \\
\hline
\end{tabular}

$n=6$ per group. Data are means \pm SEM. ${ }^{*} p<.05 ;{ }^{* *} p<.01$ vs. sham. 
Table 5. Plasma ACTH and Corticosterone (Cort) Concentrations in Response to i.v. CRH Stimulation (50 ng/kg) in rTMS-treated and Sham-treated Male HAB and LAB Rats

\begin{tabular}{lccr}
\hline & \multicolumn{3}{c}{ CRH Stimulation } \\
\cline { 2 - 4 } Line & Basal & $+\mathbf{1 0}$ Min & Delta \\
\hline LAB, sham & & & \\
$\quad$ ACTH $(\mathrm{pg} / \mathrm{ml})$ & $49.3 \pm 7.4$ & $80.9 \pm 20.1$ & $32 \pm 12$ \\
$\quad$ Cort $(\mathrm{ng} / \mathrm{ml})$ & $9.3 \pm 4.2$ & $138 \pm 12$ & $129 \pm 8$ \\
LAB, rTMS & & & \\
$\quad$ ACTH $(\mathrm{pg} / \mathrm{ml})$ & $42.1 \pm 3.5$ & $72.2 \pm 18.2$ & $30 \pm 13$ \\
$\quad$ Cort $(\mathrm{ng} / \mathrm{ml})$ & $13.1 \pm 5.2$ & $124 \pm 25$ & $111 \pm 19$ \\
HAB, sham & & & \\
ACTH $(\mathrm{pg} / \mathrm{ml})$ & $55.1 \pm 16$ & $81.9 \pm 12$ & $27 \pm 10$ \\
$\quad$ Cort $(\mathrm{ng} / \mathrm{ml})$ & $10.2 \pm 3.6$ & $135 \pm 19$ & $125 \pm 15$ \\
HAB, rTMS & & & \\
$\quad$ ACTH $(\mathrm{pg} / \mathrm{ml})$ & $48 \pm 4.1$ & $71.1 \pm 15.3$ & $23 \pm 10$ \\
Cort $(\mathrm{ng} / \mathrm{ml})$ & $15.2 \pm 8.3$ & $111 \pm 13$ & $96 \pm 8$ \\
\hline
\end{tabular}

( $n=6$ per group). No statistically significant differences between rTMS-treated and sham-treated animals.

pointing towards the specificity of the rTMS effects on stress coping behavior. Behavioral changes induced by rTMS were found to be robust, as indicated by the fact that we failed to detect any differences between naive animals and animals that had previously undergone surgery allowing for subsequent blood sampling.

\section{Neuroendocrine Effects of rTMS: Attenuation of the Stress-induced Activity of the HPA System in HAB Rats}

Disinhibition of HPA system regulation is a common feature in major depression, and clinical improvement after antidepressant treatment has been observed to be associated with a normalization of HPA system function (Holsboer and Barden 1996; Holsboer 2000). Moreover, major depression is known to be frequently accompanied by frontal cortex dysfunction (Soares and Mann 1997; George et al. 1999), whereby the frontal cortex is known to exert a regulatory role in HPA system activity (Diorio et al. 1993). Findings of blunted hormone responses to stress have been obtained in rats after chronic treatment with various antidepressants (Reul et al. 1993, 1994). Thus, since pharmacologically different drugs attenuate HPA system function, this neuroendocrine system was hypothesized to be a common denominator for clinically efficacious antidepressants (Holsboer and Barden 1996). Accordingly, changes in HPA system reactivity in human depression in response to rTMS have been suggested recently (Pridmore 1999).

Our findings on rTMS-induced changes in plasma hormone levels suggest that rTMS of frontal brain regions attenuates the stress-induced activity of the HPA system: whereas basal plasma levels of ACTH and corticosterone were almost identical in all groups, the neuroendocrine response to the ethologically relevant, combined physical and emotional stress induced by forced swimming was blunted in HAB rats. This finding is remarkable as rTMS-treated rats showed higher levels of motor activity while showing more active coping strategies. In contrast, no such effects could be observed in LAB animals. Thus, our finding supports the previously reported positive correlation between immobility in the forced swim task and plasma corticosterone levels (Báez and Volosin 1994; Korte et al. 1996). The virtually identical outcome of the $\mathrm{CRH}$ challenge test in the rTMS-treated and control HAB rats makes it unlikely that changes at the pituitary CRH-CRH 1 receptor-signaling pathway account for differences in neuroendocrine stress response. This observation, confirming previous results showing a similar response of pituitary corticotropes to a CRH challenge in HAB and LAB rats (Liebsch et al. 1998a), suggests that rTMSinduced line-specific differences in neuroendocrine regulation are likely to occur at the hypothalamic/hippocampal level. Indeed, the recent finding of a specific activation in terms of immediate-early gene expression in the paraventricular nucleus of the hypothalamus in response to rTMS supports this notion (Ji et al. 1998). Our observation of an rTMS-induced blunted HPA activity is even more interesting in light of findings suggesting that the prefrontal cortex may participate in the regulation of the neuroendocrine response to stressful stimuli and, in particular, can inhibit HPA system response to stress, i.e., CRH synthesis and release (e.g., Diorio et al. 1993). Accordingly, efferent projections of the prefrontal cortex to the perinuclear area of the hypothalamic paraventricular nucleus have been demonstrated (Hurley et al. 1991; Takagishi and Chiba 1991).

\section{Behavioral Effects of rTMS: Changes in Stress Coping Strategies}

The HAB and LAB lines, differing markedly in their inborn anxiety-related and acute stress coping behaviors (Liebsch et al. 1998a, 1998b), provide a promising tool for investigating the relationship between inborn emotionality, acute stress coping, physiological and neuroendocrine stress responses and their underlying mechanisms (Liebsch et al. 1998a). Moreover, animals genetically predisposed to certain behavioral strategies offer a unique opportunity to test therapeutic agents that are a potential treatment for abnormal behaviors, e.g., in human psychiatric disorders (Gentsch et al. 1988). Thus, studies on aberrant patterns in these selected laboratory animals may contribute to a better understanding of human psychopathology states.

The behavioral performance of rats during forced swimming primarily reflects coping strategies in re- 
sponse to a novel and stressful stimulus (Armario et al. 1988; Liebsch et al. 1998a; Marti and Armario 1993). In our hands, all rTMS-treated HAB rats adopted a more active coping strategy in that they struggled significantly more than the controls, floated less and took longer to show the first floating reaction. This finding of a robust increase in active coping abilities extends earlier reports of reduced immobility in mice (Fleischmann et al. 1995) and rats (Zyss et al. 1997) after two sessions and two 5-day series of rTMS, respectively. It should be noted, however, that these effects were observed in commercially available animals that do not necessarily reflect the pathological conditions found in patients suffering from psychiatric disease states. Most importantly, stimulation patterns used were not tested to be comparable to those used under clinical conditions. In clinical studies rTMS effects most probably relate to frontal forebrain stimulation (George et al. 1999). Consequently, to reliably investigate the underlying neurobiological effects in animal models, the adoption of equivalent stimulation conditions as used in the present study is indispensable.

It is noteworthy that rTMS-treated HAB rats, originally showing a markedly passive stress coping strategy, were found to be indistinguishable from both sham- and rTMS-treated LAB rats. This rTMS-induced shift in HAB animals towards active stress coping was markedly higher than has previously been reported in "normal" Wistar rats (Zyss et al. 1997). Thus, we could demonstrate that rTMS-induced effects are not only present in the HAB rat line but are even amplified in the genetically predisposed animal model. In contrast, rTMS-treated LAB animals that innately display rather active stress coping abilities (Liebsch et al. 1998a, 1998b) were unaffected. Our finding that rTMS differentially affected the coping abilities of $\mathrm{HAB}$ and LAB rats indicates that these treatment-induced changes are determined by the rats' innate emotionality and coping strategy. Consequently, it is tempting to extrapolate the results obtained in the present study to the clinicopathological condition. Indeed, it should be emphasized that antidepressant treatment strategies such as psychopharmacological agents exert marked beneficial actions in depressed individuals only, but not in healthy controls (Holsboer 1995; Holsboer and Barden 1996).

The occurrence of changes toward a more active coping strategy during exposure to forced swimming has frequently been shown to predict the antidepressant efficacy of a drug when administered to patients suffering from depression (Borsini and Meli 1988; Lucki 1997). Therefore, the effects of rTMS reported here point towards a potential antidepressant efficacy of this treatment. Interestingly, the extracellular concentration of dopamine, but not serotonin or noradrenaline, was found to be elevated in hippocampal microdialysates after rTMS (Keck et al. 2000). Therefore, the dopaminergic system might be one of the primary candidate neurotransmitter/neuromodulator systems that are directly affected by rTMS. In support of this hypothesis are a number of findings showing dopamine agonists to reduce floating time in rats tested in the forced swim paradigm (Borsini and Meli 1988). Furthermore, we recently reported that rTMS induced an increase in the expression of neuropeptide CCK mRNA after chronic treatment in rats (Müller et al. 2000). CCK, acting as a neuromodulator, increases the firing rate of dopaminergic ventral tegmental and substantia nigra neurons, and the ability of CCK to directly affect local dopamine release has been demonstrated in numerous experiments (for review, see Crawley and Corwin 1994). Therefore, the increase in CCK expression reported could possibly contribute to the behavioral effects of rTMS measured in the forced swim test. A further explanation for the increase in active stress coping comes from our recent finding of a significant increase in BDNF mRNA and protein in specific areas of the rat brain after chronic rTMS (Müller et al. 2000). In line with this finding is the observation that local infusion of high concentrations of BDNF into the midbrain exerts antidepressant-like effects in the forced swim test.

\section{rTMS: Differential Effects on Anxiety-related Behavior and Coping Abilities}

In contrast to $\mathrm{HAB}$ rats, rTMS-treated $\mathrm{LAB}$ rats were more anxious than their respective sham-treated controls. Accordingly, the rTMS-treated LAB animals displayed an increased stress-induced ACTH release in response to EPM exposure. This finding further supports the notion that rTMS exerts its behavioral and neuroendocrine effects differentially, depending on the basal level of emotionality. Taken together with the observation that rTMS improved stress coping abilities in HAB rats, but not in LAB rats, the present data suggest that anxiety and coping strategies may be regulated independently via different mechanisms. Further evidence for this suggestion emerges from recent findings with benzodiazepine treatment, which failed to affect the rats' overall pattern of acute stress coping in the forced swim test but had a pronounced anxiolytic effect in HAB rats (Liebsch et al. 1998a). Furthermore, the clear differences in the effects of benzodiazepines and rTMS in the HAB and LAB animals suggest that these rat lines may be a valuable tool for differentiating between predominantly anxiolytic and predominantly antidepressant effects of potential therapeutic strategies.

In conclusion, the passive coping style displayed by $\mathrm{HAB}$ rats in an acute stress situation can be turned into an active coping behavior by rTMS. Our findings herewith demonstrate that this behavioral trait, though genetically determined (Liebsch et al. 1998a, 1998b), can 
be overcome by rTMS treatment appropriately adjusted to stimulate frontal brain regions. In addition to the behavioral effects of rTMS, we present the first evidence for neuroendocrine effects in an animal model. The effects observed were present in HAB rats only, thus revealing that treatment-induced changes are determined by the rats' basal emotionality and coping strategy. Therefore, the current data could be extrapolated to the clinical condition and provide new insights into the mechanisms underlying the antidepressant effects of rTMS. With respect to the changes in HPA system activity, the present findings further support the concept of a common final pathway for different types of antidepressant treatment strategies (e.g., Müller et al. 2000).

\section{ACKNOWLEDGMENTS}

The authors would like to thank Dr. A. Curt (University Hospital Zurich, Swiss Paraplegic Centre, Zurich, Switzerland) for fruitful discussion of the experimental protocol, G. Kohl for expert handling of the RIAs, and P. Lörscher for skillful technical assistance. The magnetic stimulation device was kindly provided by DANTEC, Skovlunde, Denmark. This project is supported by the German Federal Research Ministry within the promotional emphasis "Competence Nets in Medicine" (Kompetenznetz Depression \& Suizidalität; subproject 4.5; MEK).

\section{REFERENCES}

Armario A, Galvada A, Marti O (1988): Forced swimming test in rats: Effect of desipramine administration and the period of exposure to the test on struggling behaviour, swimming, immobility and defecation rate. Eur J Pharmacol 158:207-212

Báez M, Volosin M (1994): Corticosterone influences forced swim-induced immobility. Pharmacol Biochem Behav 49:729-736

Borsini F, Meli A (1988): Is the forced swimming test a suitable model for revealing antidepressant activity? Psychopharmacology 94:147-160

Cerri G, De Leo R, Moglie F, Schiavoni A (1995): An Accurate 3-D model for magnetic stimulation of the brain cortex. J Med Eng Tech 19:7-16

Crawley JN, Corwin RL (1994): Biological actions of cholecystokinin. Peptides 5:731-755

De Bellis MD, Gold PW, Geracioti TD Jr., Listwak SJ, Kling MA (1993): Association of fluoxetine treatment with reductions in CSF concentrations of corticotropinreleasing hormone and arginine vasopressin in patients with major depression. Am J Psychiatry 150:656-667

Diorio D, Viau V, Meaney MJ (1993): The role of the medial prefrontal cortex (cingulate gyrus) in the regulation of hypothalamic-pituitary-adrenal response to stress. J Neurosci 13:3839-3847

Fleischmann A, Prolov K, Abarbanel J, Belmaker RH (1995): The effect of transcranial magnetic stimulation of rat brain on behavioral models of depression. Brain Res 699:130-132
Gentsch C, Lichtsteiner M, Feer H (1988): Genetic and environmental influences on behavioral and neurochemical aspects of emotionality in rats. Experientia 44:482-490

George MS, Lisanby S, Sackeim HA (1999): Transcranial magnetic stimulation. Arch Gen Psychiatry 56:300-311

Geyer MA, Markou A (1995): Animal models of psychiatric disorders. In Bloom FE, KupferDJ (eds), Psychopharmacology: The Fourth Generation of Progress, New York, Raven Press, pp 787-798

Greene EC (1963): Anatomy of the Rat. New York, Hafner.

Heuser IJ, Schweiger U, Gotthardt U, Schmider J, Lammers CH, Dettling M, Holsboer F (1996): Pituitary-adrenal system regulation and psychopathology during amitryptiline treatment in elderly depressed patients and in normal controls. Am J Psychiatry 153:93-99

Holsboer F (2000): The corticosteroid receptor hypothesis of depression. Neuropsychopharmacology 23:477-507

Holsboer F (1995): Neuroendocrinology of mood disorders. In Bloom FE, Kupfer DJ (eds), Psychopharmacology: The Fourth Generation of Progress, New York, Raven Press, pp 957-968

Holsboer F, Barden N (1996): Antidepressants and hypothalamic-pituitary-adrenocortical regulation. Endocr Rev 17:187-205

Hurley KM, Herbert H, Moga MM, Saper CB (1991): Efferent projections of the infralimbic cortex of the rat. J Comp Neurol 308:249-276

Ji RR, Schlaepfer TE, Aizenman CD, Epstein CM, Qiu D, Huang JC, Rupp F (1998): Repetitive transcranial magnetic stimulation activates specific regions in rat brain. Proc Natl Acad Sci USA 95:15635-15640

Keck ME, Sillaber I, Ebner K, Welt T, Toschi N, Kaehler ST, Singewald N, Philippu A, Elbel GK, Wotjak CT, Holsboer F, Landgraf R, Engelmann M (2000): Acute transcranial magnetic stimulation of frontal brain regions selectively modulates the release of vasopressin, biogenic amines and amino acids in the rat brain. Eur J Neurosci 12:3713-3720

Korte SM, De Kloet ER, Buwalda B, Bouman SD, Bohus B (1996): Antisense to the glucocorticoid receptor in hippocampal dentate gyrus reduces immobility in forced swim test. Eur J Pharmacol 301:19-25

Landgraf R, Wigger A, Holsboer F, Neumann ID (1999): Hyperreactive hypothalamo-pituitary-adrenocortical (HPA) axis in rats bred for high anxiety-related behavior. J Neuroendocrinol 11:405-407

Liebsch G, Linthorst ACE, Neumann ID, Reul JMHM, Holsboer F, Landgraf R (1998a): Behavioral, physiological, and neuroendocrine stress responses and differential sensitivity to diazepam in two Wistar rat lines selectively bred for high and low anxiety-related behavior. Neuropsychopharmacology 19:381-396

Liebsch G, Montkowski A, Holsboer F, Landgraf R (1998b): Behavioural profiles of two Wistar rat lines selectively bred for high or low anxiety-related behavior. Behav Brain Res 94:301-310

Lucki I (1997): The forced swimming test as a model for core and component behavioral effects of antidepressant drugs. Behav Pharmacol 8:523-532

Marti J, Armario A (1993): Effects of diazepam and desipramine in the forced swimming test: influence of 
previous experience with situation. Eur J Pharmacol 236:295-299

Müller MB, Toschi N, Kresse AE, Post A, Keck ME (2000): Long-term repetitive transcranial magnetic stimulation increases the expression of brain-derived neurotrophic factor and cholecystokinin mRNA, but not neuropeptide tyrosine mRNA in specific areas of rat brain. Neuropsychopharmacology, 23:205-215

Pascual-Leone A, Rubio B, Pallardo F, Catala MD (1996): Rapid-rate transcranial magnetic stimulation of left dorsolateral prefrontal cortex in drug-resistant depression. Lancet 348:233-237

Pellow S, Chopin P, File SE, Briley M (1985): Validation of open:closed arm entries in an elevated plus-maze as a measure of anxiety in the rat. J Neurosci Methods 14:149-167

Porsolt RD, LePichon M, Jalfre M (1977): Depression: a new animal model sensitive to antidepressant treatments. Nature 266:730-732

Post A, Müller MB, Engelmann M, Keck ME (1999): Repetitive transcranial magnetic stimulation in rats: evidence for a neuroprotective effect in vitro and in vivo. Eur J Neuroscience 11:3247-3254

Pridmore S (1999): Rapid transcranial magnetic stimulation (rTMS) and normalisation of the dexamethasone suppression test (DST). Psychiatry Clin Neurosci 53:33-37
Ravazzani P, Ruohonen J, Grandori F, Tognola G (1996): Magnetic Stimulation of the nervous system. Induced electric field in unbounded, semi-infinite, spherical and cylindrical media. Annals Biomed Eng 24:606-616

Reul JMHM, Stec I, Söder M, Holsboer F (1993): Chronic treatment of rats with the antidepressant amitriptyline attenuates the activity of the hypothalamic-pituitaryadrenocortical system. Endocrinology 133:312-320

Reul JMHM, Labeur MS, Grigoriadis DE, DeSouza EB, Holsboer F (1994): Hypothalamic-pituitary-adrenocortical axis changes in the rat after long-term treatment with the reversible monoamine oxidase-A inhibitor moclobemide. Neuroendocrinology 60:509-519

Soares JC, Mann JJ (1997): The functional neuroanatomy of mood disorders. J Psychiat Res 31:393-432

Takagishi M, Chiba T (1991): Efferent projections of the infralimbic (area 25) region of the medial prefrontal cortex in the rat: an anterograde tracer PHA-L study. Brain Res 566:26-39

Willner P, Muscat R, Papp M (1992): Chronic mild stressinduced anhedonia: a realistic animal model of depression. Neurosci Biobehav Rev 16:525-534

Zyss T, Gorka Z, Kowalska M, Vetulani J (1997): Preliminary comparison of behavioral and biochemical effects of chronic transcranial magnetic stimulation and electroconvulsive shock in the rat. Biol Psychiatry 42:920-924 